\title{
Agriculture biologique : Nécessité d'appropriation par les producteurs et attitudes des consommateurs au Moungo-Cameroun
}

\author{
Nathalie Lando Loyem ${ }^{1}$; Nobert Tohnain Lengha (Ph.D) ${ }^{2}$; Christopher Tankou Mubeteneh \\ (Ph.D) ${ }^{3}$ \\ 1, 2Département de Socio-Economie Rural et de Vulgarisation Agricole, ${ }^{1}$ nathalielando19@gmail.com \\ 2tohnole@yahoo.com \\ ${ }^{3}$ Department of Crop Science, Research Unit of Genetics, Biotechnology, Agriculture and plant \\ physiology, cmtankou@yahoo.com \\ ${ }^{1,2,3}$ Faculté d'agronomie et des Sciences Agricoles, Université de Dschang
}

\section{RÉSUMÉ}

L'agriculture biologique $(\mathrm{AB})$ est l'un des moyens qui contribue à répondre à certains enjeux et défis du moment en termes de sécurité alimentaire et de protection de la biodiversité, de la santé du producteur et celle du consommateur, contrairement à l'agriculture conventionnelle (AC). L'objectif de cette étude est d'analyser le comportement qu'affichent les producteurs d'ananas et les consommateurs vis-à-vis de l'appropriation de l'AB et la consommation de ses produits en vue de préserver la biodiversité. Une enquête réalisée par le biais de trois types de questionnaires différents a permis de toucher les producteurs d'ananas biologiques et conventionnels (111) et les consommateurs (318). Cette enquête a été suivie d'un guide d'entretien (7). Elle a été menée dans le département du Moungo au Cameroun ( $\mathrm{N}=436$ soit: 301 hommes et, 135 femmes; âgés entre 25 à 64 ans) plus précisément à Loum, Njombé-Penja et Mbanga. Pour mesurer l'attitude, l'étude a eu recours au modèle de Likert (1932) sous 5 échelles. La Variance et le Test de corrélation ont permis de conclure que, la protection de la biodiversité dépend majoritairement du comportement des producteurs et des consommateurs.

Mots clés : Agriculture biologique, consommateurs, attitudes, producteurs

\begin{abstract}
Organic agriculture $(\mathrm{OA})$ is a powerful means to address some of the current issues and challenges in terms of food security and biodiversity protection, producer and consumer health, unlike conventional agriculture (CA). The objective of this study was to analyze the attitudes of pineapple farmers and consumers towards the appropriation of $\mathrm{OA}$ and the consumption of its products in order to preserve biodiversity. A survey conducted using three different types of questionnaires involved 111 organic and conventional pineapple farmers and 318 consumers as well as 7 maintenance guides (7). The study was conducted in the Moungo Division,) more precisely in Loum, Njombé-Penja and Mbanga Cameroon (N = 436: The study population included 301 men and, 135 women; aged between 25 and 64 years. To measure attitude, we used Likert's model (1932) under 5 scales. The Variance and the Correlation Test suggested that biodiversity protection depends mainly on the behaviour of farmers and consumers.
\end{abstract}

Key words: Organic farming, consumers, attitudes, farmers

Received: 14/02/2020

Accepted: 24/07/2020

DOI: https//dx.doi.org/10.4314/jcas.v16i1.3

(C) The Authors. This work is licensed under the Creative Commons Attribution 4.0 International Licence. 


\section{Introduction}

«(...) Nous sommes passées d'une nourriture variée, naturelle et abondante à des produits alimentaires standardisés, industriels et uniformisés, poison pour l'homme et la nature. (...) si l'on veut prendre en main sa propre alimentation, manger sain et bon et sauver la nature, dont dépend la survie de l'humanité [il faut passer par un réveil de conscience des consommateurs et un changement de mentalité commune]» (Attali, 2019). Les pesticides entrent au Cameroun vers 1944 (Kuete, S.d.). En effet, les producteurs au Cameroun ont vu venir un nouveau vent en agriculture, présenté comme une formule magique, multipliant le rendement : les produits chimiques (engrais, pesticides, herbicides, produits de synthèses) et les OGM sans oublier les scandales du glyphosate avec Monsanto et ses victimes. L'introduction de ces produits chimiques en agriculture détruit les liens sociaux et l'esprit d'initiatives des producteurs (Howard, 1943). Les produits chimiques affectent les producteurs qui les manipulent plus ou moins sans kit de protection (Djoufack, 2016). Les résidus de ces produits chimiques se trouvent dans les aliments (des fruits et légumes) produits par des techniques agricoles conventionnelles (Phaneuf et al., 2012). Les producteurs sont principalement soucieux de l'accroissement de leur rendement agricole par conséquent, ils les utilisent abusivement (Tetang \& Foka, 2008).

Les conséquences de l'utilisation des produits chimiques en agriculture sont multiples entre autres: la perturbation de la faune, la détérioration des sols, la contamination des eaux, l'altération des végétaux et de la biodiversité. Les producteurs et les consommateurs, ne sont pas épargnés des méfaits des produits chimiques (Lieve, 2005). On le sait, les consommateurs africains prennent très peu au sérieux la notion de la qualité des produits. Ce sont en majorité les femmes et les jeunes qui s'y intéressent (Sahota, 2018). La seule satisfaction qui leur incombe est d'ingurgiter des aliments afin de satisfaire un besoin naturel. Lorsqu'il revient de parler de consommer biologique, plusieurs l'assimilent uniquement à la consommation des fruits et légumes frais, c'est-à-dire non transformés par les industries. Dans le domaine de la médecine humaine des cas de toxicité aigüe, de perturbation hormonale, de cancers, d'infertilité, de déficience immunitaire, de neurotoxicité et d'atteinte au développement du fœetus sont relevés de façon accentuée (Marchand, 2019). L'environnement africain et plus précisément l'environnement camerounais se dégrade de plus en plus, et l'agriculture conventionnelle $[\mathrm{AC}]$ contribue à cette pollution. Selon Alternative Durable pour le Développement ([ADD], 2010), environ 40\% des pesticides épandus atteignent directement la plante. La grande quantité $(60 \%)$ de la dose restante touchent directement le sol, après suit l'infiltration et le ruissellement, conséquence ; ces produits toxiques se retrouvent dans les eaux de surfaces et souterraines.

Pourtant, ces produits chimiques en agriculture avaient pour but d'accroître la production, réduire la pauvreté et la faim dans le monde. Cependant, le taux de pauvreté continu de croître de 280 millions en 1990 contre 330 millions en 2012 (Banque Mondiale [BM], 2017) l'objectif visé n'a donc pas été atteint, et la biodiversité en a pris des coups. La faim s'accroît également dans le monde entier (FAO et al., 2019), selon les Nations-Unies, la recrudescence de cette dernière est due à une situation de cause à effet émanant du changement climatique dont la cause est la pollution. En 2017 cette faim avait déjà progressé, et ce pour la troisième année consécutive (2017 $=821$ millions de personnes et $2016=804$ millions de personnes) (ONU, 2018). Il ressort au vu de tous ces dégâts qu'il est judicieux de développer des stratégies afin de résoudre ces problèmes écologiques. Surtout que la survie de l'humanité dépend de la protection de l'environnement. Cette protection est quelque peu 
conditionnée par l'orientation que donne le consommateur à son alimentation; manger sain et bon (Attali, 2019). L'agriculture biologique se présente comme stratégie idoine pour pallier aux problèmes susmentionnés.

Du codex Alimentarius, (Allaire \& Bellon, 2014) aux règlements existants, de l'agriculture traditionnelle africaine, à l'agriculture biologique, le but est le même: la préservation de l'environnement (FiBL \& IFOAM-Organic International, 2018). Au Cameroun, il existe une triple typologie sur l'agriculture biologique. Cette diversité satisfait les consommateurs à savoir : une agriculture biologique certifiée respectant des normes internationales ; une agriculture biologique dite « hybride » plus entrepreneuriale; et enfin, une agriculture biologique dite « naturelle » caractérisée par la non utilisation de produits chimiques de synthèse (Bayiha, et al., 2019). Aborder le concept agriculture biologique dans sa multiplicité permet de satisfaire toutes les couches de la population surtout les plus nécessiteux. Cette technique agricole est semblable à une ancienne technique jadis pratiquée en Afrique et qui reçut un qualificatif dévalorisant d'« agriculture archaïque », ceci en omettant son caractère de durabilité pour la génération présente et future. La présente étude cadre avec le désir pour le gouvernement camerounais d'atteindre les Objectifs du Développement Durable (ODD) et pour une émergence du Cameroun à l'horizon 2035 (DSCE, 2009 : 58). Dans la présente étude, il est question de répondre aux questions suivantes : quels sont les mécanismes d'appropriation de l'AB chez les producteurs biologiques? Quelles sont les attitudes et intentions de conversions à l'AB chez les producteurs conventionnels? Et enfin, quelles sont les attitudes et intentions des consommateurs vis-à-vis de l'AB, de l'AC et de leurs produits?

La nécessité d'une appropriation de l'AB se présente comme une alternative pour résoudre les problèmes causés par l'AC. N'est-il pas nécessaire de contribuer à la préservation de l'environnement en analysant le comportement des producteurs et des consommateurs vis-à-vis de l'AB, les principaux acteurs de la production et de la demande? L'appropriation renvoie selon Breton et Proulx à la «maitrise cognitive et technique d'un minimum de savoir-faire permettant éventuellement une intégration significative et créative de celle-ci dans la vie quotidienne de l'individu et de la collectivité » (2002: 270). Selon la présente étude, c'est le fait de prendre pour soi un objet bien défini. C'est l'intégration de l'objet dans les pratiques quotidiennes grâce à un usage répété, tout en l'adaptant au besoin réel, ce qui débouche à l'autonomisation de l'usager qui, ayant fait corps avec l'objet le transmet autour de lui. C'est également le fait de saisir fortement et intrinsèquement un objet dont on est convaincu de l'utilité afin de se l'approprier, tout en le récréant, le réinventant si possible et en lui attribuant des significations propres et ainsi mieux le valoriser et le transmettre. Elle peut dépendre des attitudes de l'individu qui conditionnent le comportement observable. Thomas et Znaniecki (1918) sont à l'origine de l'étude du concept d'attitude. Elle est définie en psychologie sociale comme un état mental et neuropsychologique de préparation de l'action, organisée à la suite de l'expérience, et qui concerne une influence dynamique sur le comportement de l'individu visà-vis de tous les objets et de toutes les situations qui s’y rapportent (Ajzen \& Fishbein, 2000). Cette théorie servira de moyen d'analyser de l'état mental des producteurs et consommateurs vis-àvis de l'agriculture biologique, de l'agriculture conventionnelle et de leurs produits. L'attitude va permettre également de prédire le comportement et conduire à un changement de mentalité des acteurs. Dans la présente étude, les hypothèses formulées stipulent que : 1- les mécanismes d'appropriation de l'AB relèvent plus de l'essaimage que d'une formation appropriée. 2- Le niveau d'intention de conversion à l'AB des 
producteurs conventionnels est déterminé par leurs attitudes envers l'AB. 3- Les consommateurs sont plus enclins à aller vers les produits biologiques que ceux issus de l'AC.

\section{Matériels et Méthodes}

Les participants de cette étude sont des agriculteurs et consommateurs d'ananas biologiques et conventionnels. Ils ont été enquêtés dans la région du Littoral, le département du Moungo plus spécifiquement dans 03 de ses arrondissements à savoir : Loum, Njombé-Penja et Mbanga.

Les agriculteurs d'ananas biologiques ont été interrogés par le biais d'un questionnaire. Le questionnaire utilisé est composé d'un préambule, d'une série de 81 questions fermées, semistructurées, à choix simples et ouvertes. Le contenu du questionnaire est meublé des questions telles que : transmettez-vous cette activité à quelqu'un? De quelle manière? Par quel moyen avezvous appris l'AB?...

Le guide d'entretien directif a contribué à la réalisation cette étude $(\mathrm{N}=07)$. Car, il est très bien détaillé et permet un suivi rapide et efficace. Les thématiques abordées concernent les mécanismes d'appropriation, les mécanismes de transmission, l'agriculture biologique et quelques sous-thèmes telles que : la maîtrise technique, cognitive et la pratique de façon répétitive.

Le répondant avait connaissance de la thématique traitée, et des sous-thèmes. Ensuite suivaient les informations sur l'anonymat de ses réponses et l'échange démarrait. Les données qualitatives ont été analysées par une analyse de contenus.

Concernant les producteurs conventionnels, le questionnaire qui leur était adressé est constitué de 74 questions (fermées, ouvertes et semis ouvertes) réparties comme suit : les 09 premières questions sont fixées sur les caractéristiques du producteur, les 13 suivantes renseignent sur les fertilisants et les méthodes de contrôle des pathologies, les 21 autres informent des propriétés de l'exploitation et le reste s'articule autour de l'attitude, les motivations et l'intention à la conversion. Le modèle de mesure proposé par Likert (1932) sous 5 points: (1- fortement pas d'accord, 2- Pas d'accord, 3- neutre, 4- d'accord, 5- fortement d'accord) est utile par endroit.

- Les caractéristiques du producteur sont évaluées par une série de 9 items. Les participants doivent répondre individuellement. Il est formulé comme suit : "l'âge », « la religion », « la situation économique ».

- Les fertilisants et les méthodes de contrôle des pathologies sont évalués par une série de 13 items. Il est formulé comme suit : "Comment préserves-tu la fertilité du sol?", "Quelles catégories de fertilisants utilises-tu? »...

- Les propriétés de l'exploitation sont analysées à partir des items suivants : "Quel est ton rôle dans l'exploitation?", "quelles sont les années de ton expérience », "quelle superficie as-tu? »...

- L'attitude envers l'appropriation de l'agriculture biologique telle qu'elle est perçue par les participants est évaluée par une série. Les participants doivent répondre sur une échelle à cinq points allant de 1-(fortement pas d'accord) à 5 (fortement d'accord). Il est formulé comme suit : "l'AB donne une image positive aux producteurs», "l'exploitation biologique est plus profitable 》les rendements en $A B$ sont plus bas »...

-L'attitude vis-à-vis de l'agriculture conventionnelle telle qu'il est perçu par les participants est évaluée par une série. Il est formulé comme suit: "l'utilisation des intrants chimiques améliore les produits", "l'utilisation des produits chimiques améliore le goût des produits ", "sans l'utilisation des produits chimiques les maladies graves seront inévitables »... Les participants doivent répondre sur une échelle en cinq points allant de 
1-(fortement pas d'accord) à 5 (fortement d'accord).

La dernière partie du questionnaire évalue leurs intentions de conversion. C'est-à-dire la probabilité pour eux à s'approprier l'AB dans les cinq prochaines années, sous une échelle à 5 points : (1- très improbable, 2- improbable, 3 neutre, 4- probable, 5- très probable). Les questions sont formulées comme suit : «Avez-vous l'intention de produire biologiquement sur ton exploitation?", "D'après vous est-il possible de se convertir à l'AB?", "Quelle sera la probabilité pour que tu convertisses ton exploitation? »... Les participants doivent donner leurs opinions sur ces affirmations et les scores des six items sont additionnés pour trouver la moyenne comportementale en cas de besoin.

Les consommateurs de la présente recherche ont été enquêtés sous inspiration de l'étude de Likert (1932) conduite dans le contexte américain adapté et utilisée par Lando et Fomba (2019) dans une étude similaire. L'instrument de collecte des données a été administré collectivement. Il faut cependant relever qu'elles sont adaptées à la variable étudiée : les attitudes et les intentions d'achats des consommateurs à l'égard de l'AB et de l'AC et leurs produits. Le questionnaire est structuré comme il suit:

- L'attitude vis-à-vis de l'agriculture biologique et ses produits tels qu'il est perçu par les participants est évaluée par une série de 7 items. Les participants doivent répondre sur une échelle en cinq points allant de 1-(fortement pas d'accord) à 5- (fortement d'accord). Il est formulé comme suit: "je crois que l'agriculture biologique est bien pour la santé; les produits biologiques sont plus appétissants »...

- L'attitude vis-à-vis de l'agriculture conventionnelle et ses produits tels qu'il est perçu par les participants est évaluée par une série de 7 items. Il est formulé comme suit: "Je crois que l'agriculture conventionnelle est très bien pour la santé; je pense que consommer les produits conventionnels me donne beaucoup de satisfactions et de santé; $»$ les participants doivent répondre sur une échelle en cinq points allant de 1-(fortement pas d'accord) à 5 (fortement d'accord). La seconde partie évalue leurs intentions d'achat sous une échelle à 5 points : (1- fortement pas d'accord 2- Pas d'accord 3- neutre 4-d'accord 5- fortement d'accord) formulée comme suit : «Je prévois acheter des produits biologiques dans un futur proche »; "Je suis prêt (e) à changer mes habitudes de consommation pour acheter plus souvent des produits conventionnels »; "j'achèterai des produits biologiques »... Les participants doivent donner leurs opinions sur ces affirmations et les scores des cinq items sont additionnés pour trouver la moyenne comportementale en cas de besoin.

Les techniques d'échantillonnage employés dans cette étude étaient des techniques probabilistes basées sur la loi des probabilités c'est-à-dire des techniques aléatoires simples dans le but de donner la chance à toutes les caractéristiques de la population d'être représentatives. Et la technique non probabiliste a été utilisée pour les producteurs biologiques. Ils ont été enquêtés par boule de neige. L'analyse statistique des données à savoir les fréquences, le test de corrélation, et la variance au seuil de signification $(\alpha<0,05)$. Le logiciel SPSS V. 23 a été utilisé pour analyser des données.

\section{Résultats}

Les participants de cette étude sont des producteurs et consommateurs d'ananas biologiques et conventionnels. Ils sont constitués des producteurs biologiques : 13 hommes et 04 femmes, soit un effectif total de 17 agriculteurs. Leurs âges varient entre 25 et 64 ans; des producteurs conventionnels : 97 hommes et 12 femmes, âgés entre 20 et 64 ans; des consommateurs : 191 hommes et 119 femmes, âgés entre 15 et 65 ans. Ces participants ont été 
interrogés dans la région du Littoral, le département du Moungo plus spécifiquement dans 03 de ses arrondissements à savoir: consommateurs (Loum : 108 ; Njombé-Penja : 111 ; Mbanga : 99), producteurs (Loum : 81 ; Njombé-Penja : 24 ; Mbanga : 06) et O7 interviews pour un total de 436 enquêtés.

Appropriation de l'agriculture biologique :

Tableau 1 : Les mécanismes d'appropriation de

l'agriculture biologique

\begin{tabular}{lll}
\hline $\begin{array}{l}\text { Appropriation de } \\
\text { l'AB }\end{array}$ & Moyenne & Écart-type \\
\hline Essaimages/Mimétisme & 4,33 & 1,53 \\
Observation & 2,67 &, 58 \\
Formation appropriée & 3,33 & 1,16 \\
Contamination & 3,00 & $\cdot$ \\
Total & 3,40 & 1,17 \\
\hline
\end{tabular}

Parmi les mécanismes d'appropriation de l'agriculture biologique présentés dans le tableau ci-dessus, il découle que les agriculteurs biologiques signalent s'être appropriés l'AB de différentes méthodes à savoir : par contamination $(\mathrm{M}=3,00 \quad ; \mathrm{E}-\mathrm{T}=0) \quad$; par formation appropriée c'est-à-dire dans un centre $(\mathrm{M}=3,33$ ; $\mathrm{E}-\mathrm{T}=1,16)$; par simple observation $(\mathrm{M}=$ 2,67 ; $\mathrm{E}-\mathrm{T}=0,58)$ et enfin par l'essaimage ou mimétisme $(\mathrm{M}=4,33$; $\mathrm{E}-\mathrm{T}=1,53)$. Il est à noter que, c'est l'essaimage qui a une moyenne plus élevée, car, c'est le moyen le plus utilisé par les agriculteurs dans le but de s'approprier de l'AB.
Il est à noter que le mécanisme Contamination et observation sont tous des éléments de l'essaimage dont $(M=10)$ l'essaimage est largement au-dessus de tout autre mécanisme. Ce mécanisme est pratiqué de façon informelle. Il résulte assurément de l'absence ou de la rareté des centres de formation en $\mathrm{AB}$ au Cameroun. Il est certes vrai que dans les écoles de formation agricole, des unités relatives à l'agriculture biologique existent, mais elles sont insuffisantes pour déclencher l'appropriation de l'agriculture biologique par un grand nombre d'agriculteurs. Voici les témoignages enregistrés lors des entretiens avec les agriculteurs :

«Nous avons eu un certain Mr Appolinaire paix à son âme; il nous a appris à faire l'AB; nous avons pratiqué plusieurs filières ».

«J'ai rencontré une maman à moi, ma'a Eli, elle faisait dans le bio, elle a tenu ma main dans ça avec beaucoup de conseils et à force de cela, j'ai pris goût».

"Je tire mon petit là dans le bio, comme je comprends, il a la volonté de faire le bio. Je lui apprends comment faire ainsi il pourra protéger aussi la nature ».

«Je serais très ravi qu'un de mes proches pratique l'agriculture biologique».

\section{Comportement planifié des producteurs conventionnels}

Tableau 2: Le comportement des producteurs conventionnels

\begin{tabular}{|c|c|c|c|}
\hline & & 1 & 2 \\
\hline \multirow{8}{*}{ 1- Attitude des producteurs conventionnels } & Corrélation de Pearson & 1 & \multirow{7}{*}{1} \\
\hline & Sig. (bilatérale) & & \\
\hline & Somme des carrés et produits croisés & 6654,49 & \\
\hline & Covariance : & 70,05 & \\
\hline & $\mathrm{N}$ & 96 & \\
\hline & Corrélation de Pearson &, $22^{*}$ & \\
\hline & Sig. (bilatérale) & 0,04 & \\
\hline & Somme des carrés et produits croisés & 330,47 & 355,33 \\
\hline \multirow{2}{*}{ 2- Intentions des producteurs conventionnels à pratiquer l'AB } & Covariance : & 3,55 & 3,74 \\
\hline & $\mathrm{N}$ & 94 & 96 \\
\hline
\end{tabular}

*. La corrélation est significative au niveau 0,05 (bilatéral). 
Il y a une corrélation significative entre l'attitude et l'intention chez les producteurs conventionnels. En effet, alpha est inférieur à 0,05 et le coefficient est compris entre 0 et $1(0,22)$. Les données des interviews, il ressort ces propos :

" L'agriculture biologique est bien je le sais mais on n'a pas de soutien du gouvernement. »

«Avant nous voyons nos ancêtres cultiver sans les produits chimiques, mais on se demande si on peut réussir l'ananas sans produits chimiques» «Nous aimons bien manger les aliments qui ne contiennent pas de produits chimiques mais pour vendre et avoir beaucoup d'argent on doit faire comment. »
«Si le gouvernement nous soutien on va se lancer dedans parce que c'est bon vraiment» «Ah! Moi je veux bien me convertir en agriculteur bio mais il faut que je sois sûre que ça rapporte » "Je produis avec les produits chimique uniquement pour vendre hein! le bio est bon j'ai mon champ bio loin d'ici et là-bas c'est pour ma famille et moi. »

\section{Attitudes et intentions d'achats chez les} consommateurs

\begin{tabular}{|c|c|c|c|c|c|c|}
\hline & & $\begin{array}{l}\text { Somme des } \\
\text { carrés }\end{array}$ & ddl & $\begin{array}{l}\text { Carré } \\
\text { moyen }\end{array}$ & $\mathrm{F}$ & Sig. \\
\hline \multirow{2}{*}{$\begin{array}{c}\text { Attitude envers l'agriculture } \\
\text { biologique } *\end{array}$} & Inter-groupes (Combinée) & 1754,95 & 30 & 58,49 & 1,81 & ,007 \\
\hline & Intra-groupes & 9266,29 & 287 & 32,29 & & \\
\hline \multicolumn{7}{|l|}{ Attitude envers l'agriculture } \\
\hline conventionnelle & Total & 11021,25 & 317 & & & \\
\hline
\end{tabular}

Il existe une différence de variance $\mathrm{F}$ entre l'attitude envers l'agriculture biologique et ses produits et l'attitude envers l'agriculture conventionnelle et ses produits. Cette différence significative est en faveur de l'agriculture biologique (F (1 318) = 1,81; $\mathrm{p}>.05)$.

Tableau 4 : Comparaison des intentions d'achats des consommateurs

\begin{tabular}{|c|c|c|c|c|c|c|}
\hline & & $\begin{array}{l}\text { Somme des } \\
\text { carrés }\end{array}$ & $\mathrm{ddl}$ & $\begin{array}{l}\text { Carré } \\
\text { moyen }\end{array}$ & $\mathrm{F}$ & Sig. \\
\hline $\begin{array}{c}\text { Intention d'achat des } \\
\text { produits biologiques } *\end{array}$ & Inter-groupes (Combinée) & 10330,89 & 54 & 191,31 & 2,72 & ,000 \\
\hline $\begin{array}{l}\text { Intention d'achat des } \\
\text { produits conventionnels }\end{array}$ & Intra-groupes & 18243,12 & $\begin{array}{l}259 \\
313\end{array}$ & 70,44 & & \\
\hline
\end{tabular}

Il existe une différence de variance $\mathrm{F}$ entre l'intention d'achat des produits biologiques et l'intention d'achat des produits conventionnels. Cette différence est en faveur de l'intention relative à l'achat des produits biologiques chez les consommateurs $\left(\mathrm{F}\left(\begin{array}{ll}2 & 313\end{array}\right)=2,72 \quad ; \mathrm{p}>.05\right)$. 


\section{Discussion}

L'objectif premier était de recenser les mécanismes d'appropriation de l'AB chez les producteurs d'ananas biologiques. Il est à noter que parmi tous les mécanismes à savoir l'observation, la contamination, la formation appropriée, c'est l'essaimage qui a la moyenne la plus élevée, car, c'est le moyen le plus utilisé par les producteurs dans le but de s'approprier de l'AB bien qu'il soit ce moyen est informel. Dans les détails et en accord avec les modèles utilisés pour transmettre les connaissances en milieu rural, les techniques de vulgarisation agricole sont multiples (Achancho \& Lothoré, 2008). Le milieu agricole a connu le système de "Training and Visit », le système de "Farmer Field School, FFS », le système d' "aide à la décision » aux exploitations familiales, et aux organisations d'agriculteurs et paysans (OP) et le programme ACEFA. Malgré l'historique d'accompagnement agricole existant, malgré les défis écologiques de l'heure, les agriculteurs transmettent l'agriculture biologique par des techniques qui leurs sont propres (Najwa, 2018). L'étude de Chatué et al. (2014) présente l'essaimage comme une «tache d'huile». Ces travaux mentionnent l'essaimage comme une tâche d'huile qu'une personne physique ou morale peut laisser dans l'esprit d'une autre. En effet, les agriculteurs biologiques optent majoritairement pour les moyens de transmissions informels, et utilisent des sources d'informations diverses pour faire passer leurs savoirs et savoirfaire. Le facteur essaimage associé à la contamination et l'observation est plus significatif (60\%), les agriculteurs biologiques ont avoué s'être appropriés l'AB par l'essaimage. Les mécanismes informels ont prévalu dans l'étude (70\%). Cette illustration pose à suffisance une question d'organisation dans le domaine, car l'AB est encore embryonnaire au Cameroun (MINEPAT, 2018). L'éducation peut être un canal idéal pour un changement de mentalité surtout que la transmission en fait, est plus large que l'enseignement, elle prend en compte des formes moins institutionnelles (Margolinas, 2016).

L'objectif second était de visualiser le lien entre les attitudes et les intentions de conversion à l'AB chez producteurs conventionnels. Il ressort à cet effet que leurs intentions sont déterminées par leurs attitudes envers l'AB. Contrairement aux résultats de Padel et Lampkin (1994), qui révèlent plutôt la disponibilité de l'information, la présence d'un expert, la présence du marché. Son originalité réside dans le fait qu'elle touche un aspect nouveau au Cameroun, particulièrement dans le département du Moungo. Ceci est contraire à certains résultats obtenus en Occident qui révèlent que les femmes sont plus enclines à la conversion en $\mathrm{AB}$ que les hommes (Burton et al., 1997 ; Burton et al., 1997). Midmore et al. (2001) affirment que plusieurs producteurs, malgré le fait qu'ils apprécient l'agriculture biologique, tardent à la conversion à cause du manque d'appui, la présence de la certification et des standards existants. Pourtant, dans cette étude, ils sont conscients de l'influence de leurs activités sur la nature et la nécessité de prendre en compte l'étude du sol avant toute entreprise (Yerima, 2013). Les résultats vont dans le même sens que ceux de Lieve (2005), qui montrent que l'attitude envers l'agriculture biologique, l'attitude de la perception de l'environnement social, et la perception de la faisabilité de la production biologique prédisent significativement les intentions des producteurs conventionnels.

L'objectif troisième était d'analyser le lien qui existe entre les attitudes et les intentions d'achats des consommateurs. Il ressort dans un premier temps que ces derniers sont plus enclins à aller vers les produits biologiques que ceux issus de l'agriculture conventionnelle et entre autres leurs attitudes prédisent mieux leurs intentions d'achats. En effet, les consommateurs ont manifesté plus d'intention d'achat envers les 
produits biologiques que conventionnels. Ils sont plus influencés par des facteurs tels que : les besoins de santé (plus accentué), la croyance et convictions personnelles, la protection environnementale, la croyance et la détermination personnelle, les raisons de confiance, de disponibilité, de pouvoir économique, d'aisance, de pouvoir économique et d'une grande disponibilité, pour des motifs de changement d'habitude alimentaire et afin pour se conformer à la mode. Contrairement aux résultats de Padel et Lampkin (1994), qui révèlent plutôt la disponibilité de l'information, la présence d'un expert, la présence du marché. Son originalité réside dans le fait qu'elle touche un aspect nouveau au Cameroun, particulièrement dans le département du Moungo. Ceci est contraire à certains résultats obtenus en Occident qui révèlent que les femmes sont plus enclines à la conversion en $\mathrm{AB}$ que les hommes (Burton et al., 1997, Burton et al., 1997). Ces résultats vont dans le même sens que ceux de Lieve (2005), qui montrent que l'attitude envers l'agriculture biologique, l'attitude de la perception de l'environnement social, et la perception de la faisabilité de la production biologique prédisent significativement les intentions des producteurs conventionnels. Le fait que les consommateurs sont désormais informés influencent la formation de leurs attitudes «mais chez les consommateurs on note une toxicité aigüe, la perturbation hormonale, les cancers, l'infertilité, les déficiences immunitaires, la neurotoxicité et l'atteinte au développement du fœutus chez la mère (Marchand, 2019) ». Ces résultats ne corroborent pas ceux de Wang et al. (2019) qui relèvent que les normes subjectives, sont de bons prédicteurs d'intention d'achat des produits biologiques en Tanzanie et au Kenya. Cette étude est également divergente à celle de Deloitte (2015), qui révèle que les consommateurs préfèrent plus les marques des produits alimentaires locaux au détriment des marques internationales et ceux n'ayant aucune marque ou inconnue. En plus, le comportement individuel peut être motivé par l'affection qui doit d'abord être décidée ou planifié (Karim, 2018). Ces résultats convergent à ceux de Lando (2013) qui relevait également que le niveau d'intention d'achat des consommateurs est déterminé par l'attitude de ces derniers envers le haricot vert et le haricot beurre. En plus, il est à noter dans cette étude que la valorisation et la production des produits locaux biologiques favorisent une appropriation majoritaire, qui permet de vivre dans un environnement favorable à la saine alimentation (Landriault, et al., 2019).

\section{Références}

Achancho, V. et Lothoré, A. (2008). Dispositifs de vulgarisation et conseil agricole au Cameroun : vers la reconnaissance par les politiques agricoles des organisations de producteurs et des exploitations familiales. Groupe de Travail Thématique inter réseaux Développement rural « service agricole ». p. 7-10.

Ajzen, I., and Fishbein, M. (2000). "Attitudes and the attitude-behaviour relation: Reasoned and automatic processes", in Stroebe, W. and Hewstone, M. (Eds.), European Review of Social Psychology, Chichester, England, Wiley 11, pp. 133

Allaire G. et Bellon S. (2014). L'AB en 3D : diversité, dynamiques et design (ou dessein), de l'agriculture biologique. Working paper. Africa, Nations Unies, New York Et Genève, 2.

Alternative durable pour le développement [ADD]. (2010).

Attali, J. (2019). Histoire de l'alimentation. Librairie Arthème Fayard Adagp. 
Banque Mondiale [BM]. (2017). Sécurité alimentaire et nutritionnelle. https:// www.banquemondial.org

Bayiha G. D. L. P. et al. (2019). Typologie et perspective d'évolution de l'agriculture biologique au Cameroun. Cah. Agric. 28 : p. 3.

Bioret, F.et al. (2009). Le dictionnaire de la protection de la nature. Presses Universitaires (PUR).

Breton, Ph. et S. Proulx. (2002). «Usages des technologies de l'information et de la communication ». In L'Explosion de la communication à l'aube du XXIe siècle. Montréal : Boréal. 251-276.

Burton, M. et al. (1997a). Sustainable agriculture technologies: A quantitative analysis of adoption. Paper presented at the Agricultural Economics Society Annual Conference, Edinburgh. P. 5-11.

Burton, M. et al. (1997b). Why do UK organic horticultural producers adopt organic techniques? NENOF (6) pp. 7-10.

Chatué, J. et al. (2014). Qu'appelle -t-on vulgariser? Éléments pour un bilan d'étapes des pratiques de vulgarisation en Afrique subsaharienne. Revue Nka' Lumière. pp. 83-105.

Deloitte. (2015). La consommation en Afrique. Le marché du XXI Siècle Éditorial.

Djoufack Tonleu, C. A. (2016). « Analyse de la place des intrants chimiques dans les activités agricoles en milieu rural: cas des maraîchères agricoles. » Session de mai, Université de Dschang Cameroun.

Document de stratégie pour la croissance et l'emploi (DSCE). (2009). Cadre de référence de l'action gouvernementale pour la période 2010-2020:58
FAO, et al. (2019). Agriculture/ Climate change and environment/ Food and Nutrition. pp. 1-6.

FiBL \& IFOAM-Organic International. (2018). The World of Organic Agriculture. Frick and Bonn.

Howard, A. 1943. An agriculture testament (PDF) Oxford, UK: oxford University Press, archived from the original on 2 july 2010, retrieved the 9th of August per special Rodale Press Eds 1976.

Kuete, M. (S.d.). Agriculture biologique au Cameroun, redécouverte ou nouvelle panacée? Université de Dschang, présentation Orale à la Faculté d'Agronomie et des Sciences Agricoles, Conférence.

Lando, L. N., et Fomba, M. E. (2019). Attitude et intention d'achat chez les consommateurs du haricot vert et du haricot beurre : cas des producteurs-Consommateurs de PROLEG.SA de Balessing. Revue Africaniste InterDisciplinaire Monange, (8). pp. 47-64

Lando, Loyem. N. (2013). Attitude et intention d'achat : le cas des consommateurs du haricot vert et beurre (les agriculteurs de proleg) de Balessing.

Landriault, J-P. et al. (2019). Intervention économique pour augmenter la consommation de fruits et légumes : étude sur la faisabilité et l'acceptabilité de l'intervention. Bibliothèque et Archives nationales $d u$ Québec. Direction $d u$ développement des individus et des communautés, Institut Nationale de Santé publique, gouvernement du Québec. 9(7). pp. 8-25.

Lieve, D. C. (2005). Determinants of organic farming conversion. Paper prepared for poster presentation at the XIth International Congress of the EAA (European Association of Agricultural Economists) 'The Future of Rural 
Europe in the Global Agri-Food System', Copenhagen, Denmark, 24-27.

Likert, R. (1933). "A technique for the measurement of attitudes» Archives of psychology, (140), pp. 1-55.

Marchand, S. (2019). Service documentaire EHESP-Sélection bibliographiques dans le cadre de la semaine sans pesticide du 20 au 30 mars 2019.

Margolinas, C. (2016). La transmission des savoirs et des connaissances. Du mot au concept: Transmission Grenoble. Hal-0188321. pp. 1-7.

Midmore, P. et al. (2001). Attitudes towards conversion to organic production systems: A study of farmers in England. Institute of rural Studies, University of Wales, Aberystwyth. p. 65.

MINEPAT, (2018). Note d'analyse prospective. L'agriculture biologique, l'agriculture de demain ? Cellule de la prospective économique au Cameroun. Note No 001/2018 Octobre.

Najwa, A. (2018). L'évolution du système de vulgarisation agricole face aux nouveaux défis de l'agriculture et aux enjeux de l'agroécologie dans les pays du Sud et de l'Est de la Méditerranée : le cas de la Syrie et de la Tunisie. Économies et Finances. Université Bourgogne. p. 33.
Padel, S. et al. (1999). Influence of policy support on the development of organic farming in the European Union. International Planning Studies 4 (3), pp. 303-315.

Phaneuf, D. et al. (2012). Étude sur la présence de résidus chimiques dans les aliments consommés au Quebec. Institut Nationale de la Santé publique. Direction de la santé environnementale et de la toxicologie. p. 18.

Sahota, A. (2018). The global Market for Organic Food \& Drink: The World of Organic Agriculture. Frick and Bonn.

Tetang, T. J. et Foka, G. (2008). Utilisation des pesticides dans la zone du Moungo-Evaluation de l'impact sur l'environnement de Njombé-Penja. https : // docplayer.fr5287066-

Thomas, W. I., et Znaniecki, F. (1918). L'attitude un concept-clé pour la compréhension des comportements. Université de Chicago

Wang, X. et al. (2019). Factors Influencing Organic Food Purchase Intention in Developing Countries and the Moderating Role of

Knowledge. Sustainability. 11 (209) p. 55. https:/ Ldoi:10.3390/su11010209.

Yerima, B. P. K. (2013). Handbook for plants manures compost and municipal waste analysis. (Eds clé) Yaoundé. pp. 26-33. 